\title{
Application of Density Corrected Spalart-Allmaras Model to Flow Past Ogive Cylinder at High Angles of Attack
}

\author{
N. $\operatorname{Kumar}^{1 \dagger}$ and M. T. Nair ${ }^{2 *}$

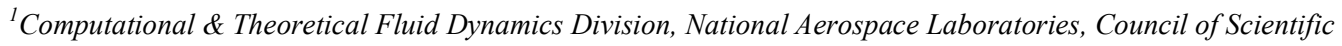 \\ \&Industrial Research, Bangalore-560017, India \\ ${ }^{2}$ Computational \& Theoretical Fluid Dynamics Division, National Aerospace Laboratories, Council of Scientific \\ \&Industrial Research, Bangalore-560017, India \\ *Currently, Associate Professor, Department of Aerospace Engineering, Indian Institute of Space and Technology, \\ Thiruvananthapuram- 695547, India" \\ $\dagger$ Corresponding Author Email: naresh@ctfd.cmmacs.ernet.in
}

(Received November 29, 2011; accepted December 25, 2012)

\begin{abstract}
Computation of flow past high speed vehicles requires the use of a reliable turbulence model. Unfortunately, most of the turbulence models are developed for incompressible flows. Application of these models directly to high speed boundary layers with large density gradients can lead to significant errors in prediction of skin friction. Several compressibility corrections have been suggested in literature to predict these turbulent flows at high Mach numbers. In the present work, we have used two such corrections for the Spalart-Allmaras turbulence model and studied their performance at high angles-of-attack. Flow past an ogive cylinder is considered for the study.
\end{abstract}

Keywords: Spalart-Allmaras, Turbulence modeling, High angle of attack, Compressibility.

\section{NOMENCLATURE}

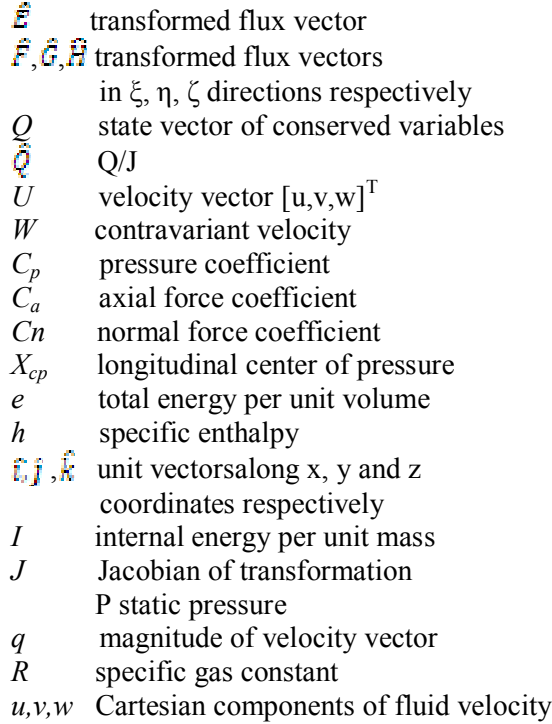

\begin{tabular}{|c|c|}
\hline$x, y, z$ & Cartesian coordinates \\
\hline$P_{r}$ & Prandtl number \\
\hline$\alpha$ & angle of attack \\
\hline$\gamma$ & $\begin{array}{l}\text { ratio of specific heats (isentropic } \\
\text { constant) }\end{array}$ \\
\hline$\kappa$ & $\begin{array}{l}\text { index representing } \xi, \eta, \zeta \\
\text { respectively }\end{array}$ \\
\hline$\lambda$ & eigenvalue \\
\hline$\rho$ & density \\
\hline $\begin{array}{l}\xi, \eta, \zeta \\
\tau\end{array}$ & $\begin{array}{l}\text { transformed (computational) coordinates } \\
\text { transformed time }\end{array}$ \\
\hline$\mu$ & coefficient of molecular viscosity \\
\hline$\mu_{t}$ & coefficient of eddy viscosity \\
\hline $\mathrm{y}^{+}$ & $\frac{p \text { a }}{\underline{\mu}}$ non-dimensional wall distance \\
\hline $\begin{array}{ll}n \\
\Delta\end{array}$ & $\begin{array}{l}\text { friction velocity } \\
\text { distance from the wall }\end{array}$ \\
\hline \multicolumn{2}{|c|}{ Subscripts } \\
\hline$i, j, \mathrm{k}$ & spatial indices \\
\hline$v$ & viscous \\
\hline$\infty$ & freestream conditions \\
\hline
\end{tabular}




\section{INTRODUCTION}

High speed flows are now routinely computed by using Computational Fluid Dynamics (CFD). Numerical computations of hypersonic flows have gained importance due to the inability of the experimental facilities to match all the conditions in flight. High Mach number flows are characterized by the presence of high density regions due to occurrence of the shocks. These shocks interact with the boundary layer present and thus complicating flow-field further. Reynolds Averaged Navier-Stokes (RANS) computation of turbulent flows at these speeds requires the turbulence model to accommodate these compressibility effects. At high speeds, shocks when contained inside the boundary layer give rise to high density gradients in the boundary layer.

Unfortunately, most of the turbulence models are developed for incompressible flows and they do not account for such high density gradients. Direct application of these turbulence models may lead to significant errors for high-speed applications. Compressibility is not considered important for wallbounded flows up to Mach numbers of 5, provided the flow doesn't experience strong shocks. Many researchers have proposed compressibility corrections to turbulence models. Most of these models either add a dilatation-dissipation term or a pressure-dilatation term to the kinetic turbulent energy (TKE) equation. For high speed turbulent mixing layers, Zeman (1990) and Sarkar et al.(1991) suggested modification to the TKE equation through the addition of a dilatation dissipation term which is a function of turbulent Mach number Mt. Sarkar (1992) and Zeman (1993) suggested a pressuredilatation term in turbulent kinetic energy equation as compressibility correction. Catris and Aupoix (2000) have suggested a correction of the diffusion terms in the two-equation models in order to make them consistent with the logarithmic law of compressible boundary layers. They also suggested similar corrections for the Spalart- Allmaras model (Spalart and Allmaras 1992). The results for flat plate case for different Mach and Reynolds number were shown in Catris and Aupoix (2000). Deck et al. (2002) have used modified version of the model for flow past ogive cylinder and for inlet flows. The aim of the present work is to study the suitability of compressibility corrections to SpalartAllmaras (SA) model for high angle of attack flows. Compressibility corrections due to Catris and Aupoix (2000) and Deck et al. (2002) have been implemented and tested for flow past an ogive- cylinder at high angles of attack for different Mach numbers and Reynolds numbers. The obtained results are compared with previous results due to the present authors Kumar et al. $(2004,2005)$ using original SA model. Also compared are the experimental and computational results of Birch et al. (1994) and Sturek et al. (1998). Birch et al. (1994) have studied laminar and turbulent flow past the ogive-cylinder using Parabolized NavierStokes (PNS) equations with Baldwin and Lomax (1978) model and Degani and Schiff(1986) correction. They studied three different cases varying the Mach number and Reynolds number. Josyula (1999) has done Reynolds Averaged Navier-Stokes (RANS) computations employing $\mathrm{k}-\varepsilon$ turbulence model along with compressible correction. Sturek et al. (1998) have studied six different cases for transonic and supersonic Mach numbers at $8^{\circ}$ and $14^{\circ}$ angles of attack.

\section{GOVERNING EQUATIONS}

The most general description of the fluid flow is provided by the time dependent compressible NavierStokes equations which express the conservation laws for mass, momentum and energy for viscous fluids. For turbulent flows, the so called Reynolds Averaged Navier-Stokes (RANS) equations are used. They are derived from the Navier-Stokes equations by introducing a time averaging procedure. The laws of motion are then expressed for the mean time averaged quantities. This makes the equations of turbulent flows look like the same as equations for laminar flow.

The RANS equations in the general, body-fitted coordinate system are given as

$\frac{\partial \phi}{\partial \tau}+\frac{\partial F}{\partial \xi}-\frac{a G}{\partial \eta}+\frac{\partial g}{\partial \eta}=\frac{1}{\operatorname{Re}}\left\{\frac{\partial F_{v}}{\partial \xi}+\frac{a \hat{G}_{v}}{\partial \eta}+\frac{a A_{v}}{\partial \xi}\right\}$

where,

$\phi=\frac{1}{J}\left(\begin{array}{c}\theta \\ \rho \\ \rho w \\ \rho w \\ \rho w\end{array}\right) \quad \vec{E}=\frac{1}{3}\left(\begin{array}{c}(e+p) W \\ \rho W \\ \rho w+p \kappa_{z} \\ \rho w W+p \kappa_{y}\end{array}\right)$

$\mathrm{Q}=\mathrm{J} \vec{\emptyset}$ is the state vector of conserved variables and $\mathrm{J}$ is the Jacobian of the transformation. E denotes an inviscid flux vector and can be $\mathbb{F}, \mathcal{G}, \mathcal{A}$ depending on $\kappa=$ $\xi, \eta, \zeta$ respectively. The contravariant velocity is given by $\mathrm{W}=\mathrm{uk}_{\mathrm{x}}+\mathrm{vk}_{\mathrm{y}}+\mathrm{wk}_{\mathrm{z}}$. The Cartesian velocity components are $\mathrm{u}, \mathrm{v}$ and $\mathrm{w}$ respectively and the velocity vector $\mathrm{U}=\mathrm{u} \hat{f}+\mathrm{v} f+\mathrm{w} \hat{k}$ with $\mathrm{q}^{2}=\mathrm{U} \cdot \mathrm{U}$. The fluid density is $\rho$. The total energy per unit volume is $e=\rho I+$ $\frac{1}{2} \rho q^{2}$ where $\mathrm{I}$ is the internal energy per unit mass of the gas. The pressure $p$ is given by the equation of state $p=$ $(\gamma-1) \rho \mathrm{I}$. Re is the free-stream Reynolds number based on a characteristic length.

$\hat{\boldsymbol{E}}_{\mathrm{v}}$ denotes a viscous flux vector and can be $\hat{F}_{v}, \hat{G}_{\mathrm{v}}, \hat{H}_{\mathrm{v}}$ depending on $\kappa=\xi, \eta, \zeta$ respectively. It is given as

$\hat{E}=\left(\frac{\kappa_{x}}{J}\right)\left(F_{v}\right)+\left(\frac{\kappa_{y}}{J}\right)\left(G_{v}\right)+\left(\frac{\kappa_{z}}{J}\right)\left(H_{v}\right)$

where $J$ is given by $J=\frac{\partial(\sin x)}{B(x y z)}$. The inverse of a Jacobian represents the volume of a cell. The metric coefficients $\frac{\pi}{j}$ etc. represent surface normals.

The Cartesian fluxes are

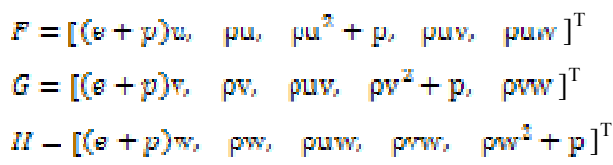




$$
\begin{aligned}
& F_{v}=\left[u \tau_{x x}+v \tau_{x y}+w \tau_{x z}-q_{x}, 0, \tau_{x x}, \tau_{x y}, \tau_{x z}\right]^{T} \\
& G_{v}=\left[u \tau_{x y}+v \tau_{y y}+w \tau_{y z}-q_{y}, 0, \tau_{x y}, \tau_{y y}, \tau_{y z}\right]^{T} \\
& H_{v}=\left[u \tau_{x z}+v \tau_{y z}+w \tau_{z z}-q_{z}, 0, \tau_{x z}, \tau_{y z}, \tau_{z z}\right]^{T}
\end{aligned}
$$

The pressure is calculated by the equation of state

$$
p=(\gamma-1)\left(e-\frac{\rho\left(u^{2}+v^{2}+w^{2}\right)}{2}\right)
$$

where $\gamma$ is the ratio of specific heats.

The elements of the shear-stress tensor and the heat flux vector are given by the equations for the Newtonian fluid

$$
\begin{aligned}
& \tau_{x x}=2 \mu u_{x}-\frac{2}{3} \mu\left(u_{x}+v_{y}+w_{z}\right) \\
& \tau_{y y}=2 \mu v_{y}-\frac{2}{3} \mu\left(u_{x}+v_{y}+w_{z}\right) \\
& \tau_{z z}=2 \mu v_{z}-\frac{2}{3} \mu\left(u_{x}+v_{y}+w_{z}\right) \\
& \tau_{x y}=\tau_{y x}=\mu\left(u_{y}+u_{x}\right) \\
& \tau_{x z}=\tau_{z x}=\mu\left(u_{z}+w_{x}\right) \\
& \tau_{y z}=\tau_{z y}=\mu\left(v_{z}+w_{y}\right) \\
& q_{x}=-\phi \frac{\partial T}{\partial x}, q_{y}=-\phi \frac{\partial T}{\partial y}, q_{z}=-\phi \frac{\partial T}{\partial z}
\end{aligned}
$$

where, $\phi=\frac{\mu}{\%-1 w_{j} z_{1}} . T$ is the static temperature and $\mu$ is the coefficient of viscosity. It may be noted that for turbulent flow, we write $\mu=\mu_{m}+\mu_{t}$ where $\mu_{m}$ is the coefficient of molecular viscosity and is a property of the fluid, while $\mu_{t}$ is called the coefficient of eddy viscosity and is a property of the flow. The coefficient of molecular viscosity is computed employing Sutherland's law and a turbulence model is used to obtain the coefficient of eddy viscosity. The free-stream Mach number is denoted by $M_{\infty}$.

Euler equations can be obtained by dropping the viscous terms.

\section{Spalart- Allmaras (SA) TURbUlenCE MOdel}

Inspired by the work due to Baldwin and Barth (1990),Spalart and Allmaras (1992) developed a new one equation model which was derived from scratch to avoid any ancestry and ensure future growth. The derivation employs certain empiricism and arguments of dimensional analysis, Galilean invariance, and selective dependence on the molecular viscosity. The model is numerically forgiving, in terms of near wall resolution and stiffness, and yields fairly rapid convergence to steady state. The wall and freestream boundary conditions are trivial. The details of the implementation and limited testing of original SA model in the code MB-EURANIUM are available in Saxena and Nair (2002). In the present work two types of compressibility corrections to SA model as given in
(Catris and Aupoix 2000 and Deck et al. 2002) are implemented as explained below.

The Spalart and Allmaras (1992) model belongs to the family of eddy viscosity models. In this model, the kinematic turbulent viscosity $v_{\mathrm{T}}$ is computed via an intermediate variable through the relation

$v_{T}=\tilde{v} f_{v 1}(\chi)$

where $\chi=\frac{\pi}{0} f_{\mathrm{v} 1}=\frac{X^{2}}{X^{2}+z_{\mathrm{v} 1}} \cdot f_{1}$ is a damping function and $\mathrm{c}_{\mathrm{v} 1}$ is a calibration constant having a value of 7.1. The intermediate variable $\theta$ is computed through the following transport equation

$$
\begin{aligned}
& \frac{D \tilde{v}}{\mathrm{Dt}}=\mathrm{c}_{\mathrm{b} 1} \tilde{S} \tilde{v}+\frac{1}{\sigma}\left[\nabla \cdot((v+\tilde{v}) \nabla \tilde{v})+\mathrm{c}_{\mathrm{b} 2}(\nabla \tilde{v})^{2}\right] \\
& -\mathrm{c}_{\mathrm{w} 1} \mathrm{f}_{\mathrm{w}}(\mathrm{r})\left(\frac{\tilde{v}}{\mathrm{~d}}\right)^{2}
\end{aligned}
$$

where $S=\mathrm{S}+\frac{w}{k^{2} d^{2}} f_{v z}(x), \mathrm{S}$ is the magnitude of vorticity, $\mathrm{d}$ is distance to the wall and $f_{\mathrm{v} 2}$ is another damping function defined as

$$
f_{v 2}(\chi)=1-\frac{\chi}{1+\chi f_{v 1}(\chi)}
$$

$f_{\mathrm{w}}$ is a function which allows faster decay of the destruction term in the outer region of the boundary layer. This function depends on the characteristic length defined by $r=\frac{w}{s k^{2} d^{2}}$. The function $f_{w}$ is defined as

$f_{w}(r)=g(r)\left[\frac{1+c_{w 3}^{6}}{g(r)^{6}+c_{w 3}^{6}}\right]^{1 / 6}$

$g(r)=r+c_{w 2}\left(r^{6}-r\right)$

$c_{b 1}, \quad c_{b 2}, \sigma, k, c_{w 1}, c_{w 2}, c_{w 3}, c_{v 1}$ are constants of the model (see Saxena and Nair 2002 and Spalart and Allmaras 1992).

1.

2.

3.

\subsection{Density Corrected SA Model}

Compressibility can have significant effect on turbulence production and also on mechanism of energy exchange between different modes. The original SA model does not take care of variation of density in the boundary layers. Catris and Aupoix (2000) have suggested a modification to the diffusion term in order to make the turbulence model consistent with the logarithmic law for compressible boundary layers. They transport $v_{\mathrm{T}}$ and diffuse $\sqrt{\rho} v_{\mathrm{T}}$ in their model.

The modified equation is written as 
N. Kumar and M. T. Nair / JAFM, Vol. 6, No. 3, pp. 375-384, 2013.

$$
\begin{aligned}
& \frac{D \rho \tilde{v}}{D t}=c_{b 1} \tilde{S} \rho \tilde{v}+\nabla \cdot\left(\frac{1}{\sigma} \mu \nabla \tilde{v}\right)+ \\
& \nabla \cdot\left(\frac{1}{\sigma} \sqrt{\rho} \nabla \nabla \sqrt{\rho} \tilde{v}\right)-c_{w} f_{w}(r) \rho\left(\frac{\tilde{v}}{d}\right)^{2}
\end{aligned}
$$

Deck et al. (2002) prefer another form of the above equation, given as

$$
\begin{aligned}
& \frac{D \rho \tilde{v}}{D t}=c_{b 1} \tilde{S} \rho \tilde{v}-\rho c_{w} f_{w}(r)\left(\frac{\tilde{v}}{d}\right)^{2} \\
& +\frac{1}{\sigma}\left[\nabla \cdot(\rho(v+\tilde{v})) \nabla \tilde{v}+c_{b 2}(\nabla \tilde{v} \nabla \rho \tilde{v})\right]
\end{aligned}
$$

\section{NUMERICAL APPROACH}

A brief outline of the numerical approach followed to solve the equations is presented here. It employs a Total Variation Diminishing (TVD) formulation based on Monotone Upwind Scheme for Conservation Laws (MUSCL) within the frame work of cell centered finite volume approach and generalized body-fitted coordinates to discretize the Euler terms. The Euler terms are discretized using the Roe's (Roe 1981) fluxsplitting method. The limiter used is due to van Albada (1982). The viscous terms are central differenced. The time term is discretized using the matrix-free LU-SSOR implicit time stepping scheme (Yoon and Jameson 1987). The Spalart- Allmaras equation is solved using a point implicit method (Saxena and Nair 2002).

\section{RESUlts AND Discussion}

The ogive-cylinder geometry is shown in Fig. 1. It is of 13 diameters length, first 3 diameters length is ogive and the rest 10 diameters length is a cylinder. The definition of the ogive is provided in the figure. Two different test cases of varying Mach number and Reynolds number considered are as shown in the Table 1. The freestream conditions are shown in the Table 2.

A view of the 10-block structured grid having 1.68 million grid points generated using GRIDGEN (Gridgen 1999) is shown in the Fig. 2. The grid consists of 151 points along the axis of the body and 101 each in the radial and circumferential directions. Thegrid is asymmetric-the domain extends to a larger distance on the leeward side compared to the windward side.

Table 1 Test Cases

\begin{tabular}{|c|c|c|c|}
\hline Case & $M_{x s}$ & $\alpha$ & $\operatorname{Re}_{\infty}$ \\
\hline 1 & 2.5 & $14^{0}$ & $1.23 \times 10^{6}$ \\
\hline 2 & 1.8 & $14^{0}$ & $0.89 \times 10^{6}$ \\
\hline
\end{tabular}

Table 2 Freestream conditions

\begin{tabular}{|c|c|}
\hline Parameter & \\
\hline Freestream pressure, $\mathrm{P}_{\infty}$ & $8300.8 \mathrm{~N} / \mathrm{m}^{2}$ \\
\hline Freestreamtemperature, $\mathrm{T}_{\infty}$ & $136.88 \mathrm{~K}$ \\
\hline Ratio of specific heats, $\gamma$ & 1.4 \\
\hline
\end{tabular}

\begin{tabular}{|c|c|}
\hline Characteristic Length & $0.09398 \mathrm{~m}$ \\
\hline Wall condition & Adiabatic \\
\hline
\end{tabular}

$\mathbf{r}(\mathbf{x})$

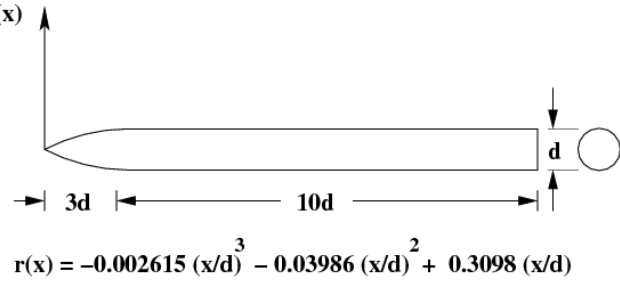

Fig. 1. Definition of the configuration

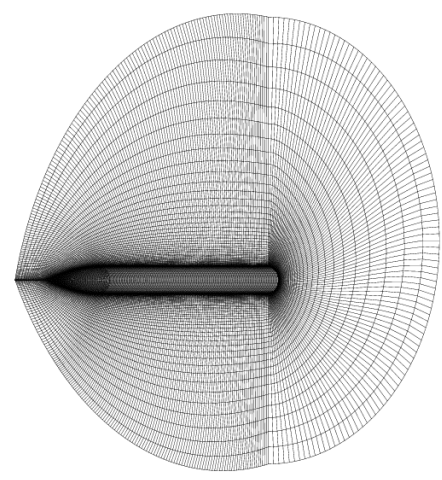

Fig. 2. Multi-block grids: $151 \times 101 \times 101$

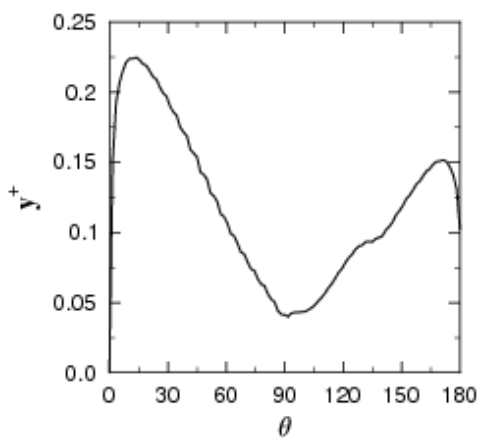

(a) Case 1

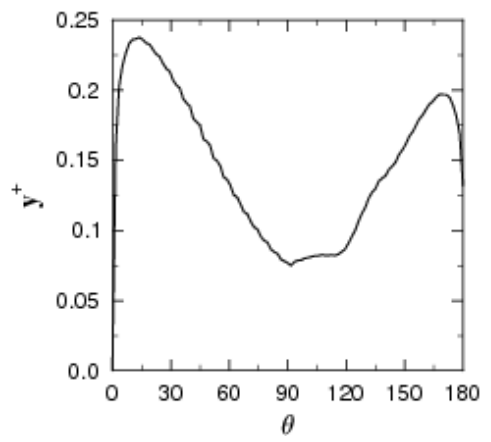

(b) Case 2

Fig. 3. $\mathrm{y}^{+}$Distribution in the circumferential directionat $\mathrm{x} / \mathrm{d}=11.5$ 
This allows for better resolution of the vortical flow on the leeward side and strong shocks on the windward side. Since no yaw angle is considered in the present computations, only half the grid in circumferential direction is used. The $\mathrm{y}^{+}$distribution along circumferential direction at $90 \%$ of the length of body is shown in the Fig. 3 for the fine grid for both cases. The $\mathrm{y}^{+}$value at the wall ranges from 0.025 to 0.225 .

The comparison between the Catris and Aupoix (2000) and Deck et al. (2002) corrections is shown in Fig. 4 at two axial locations for Case 2. The results are essentially the same for both, hence results for Catris and Aupoix (2000) corrections for Spalart-Allmaras model (SA+CA) are only discussed in the remaining of the paper. The density contours for Case 1 and Case 2 are shown in the Fig. 5. The presence of strong shocks and large separated regions are noticed on windward and leeward sides respectively.
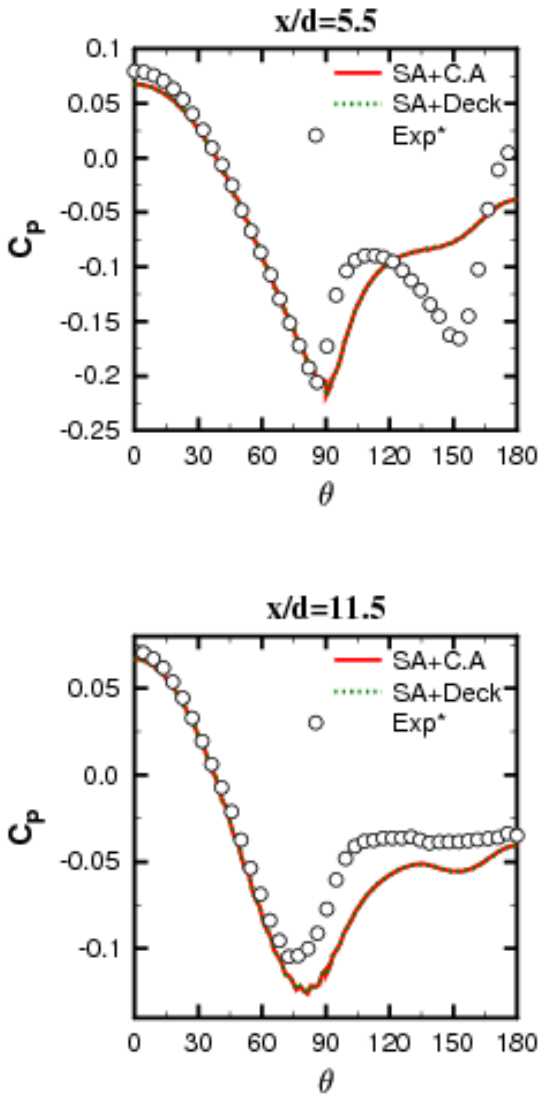

Fig. 4. Case 2: Comparison of present computed $C_{p}$ using two versions of compressibility corrections with experimental results of Sturek et al. (1998)*

The variation of coefficient of pressure along the surface, $C_{p}$ vs. $\theta$, is shown in the Fig. 6 and Fig. 7 at four different axial locations for Case 1 and Case 2 respectively.

Figure 6 shows the $\mathrm{C}_{\mathrm{p}}$ results for Case 1 plotted along with our previous results of original SA model (Kumar et al. 2004, 2005) and other experimental and computational results of Birch et al. (1994). It can be noted that the $C_{\mathrm{p}}$ curve follows closely to the experimental one till about the separation point. Prediction of the separation point is off except at $\mathrm{x} / \mathrm{d}=5.5$ for both the SA models. However, the corrected model predicts the pressure at separation point and location of separation point better in comparison to the original model. The secondary separations and associated recirculation zones are well formed for the original SA model. At $\mathrm{x} / \mathrm{d}=5.5$, the corrected model fails to capture the secondary recirculation zone. At other sections, the SA model shows stronger secondary vortices compared to experiments, whereas these vortices are weaker with $\mathrm{SA}+\mathrm{CA}$ model.

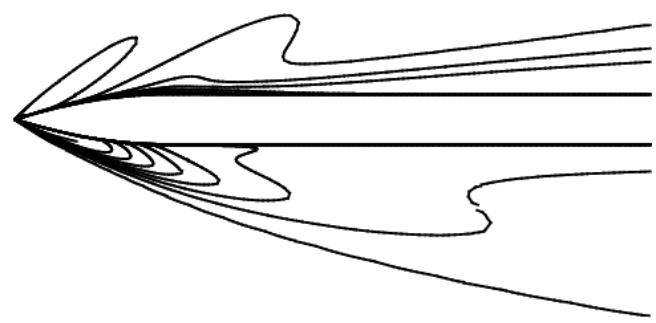

(a) Case 1: $M_{x}=2.5, \alpha=: 4^{2}$

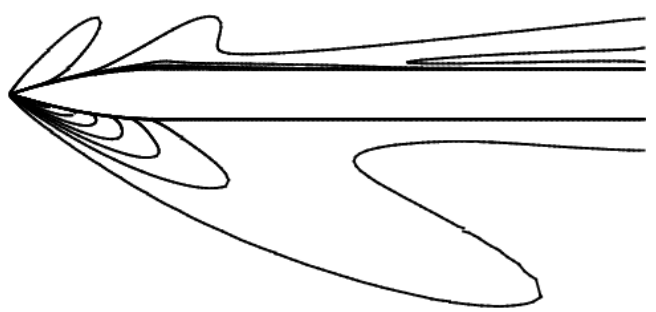

(b) Case 2: $M_{x}=1,8, \alpha=14^{2}$

Fig. 5. Pitch plane density contours

The Fig. 7 shows the comparison of coefficient of pressure for the present computations for the Case 2 with the experimental and computational results of Sturek et al. (1998) at four different $\mathrm{x} / \mathrm{d}$ stations similar to Case 1.Here again it can be noted that the corrected model works better in the attached region. The pressure at separation and the separation point location itself is better predicted with $\mathrm{SA}+\mathrm{CA}$.

The SA model shows stronger secondary separation and associated vortices when compared to the SA+CA model. The corrected models fail to capture any secondary vortex at $\mathrm{x} / \mathrm{d}=5.5$. Whereas, for both cases computations of Birch et al. (1994) (for Case 1 ) and Sturek et al. (1998) (for Case 2 ) using the Baldwin Lomax model along with Degani and Schiff correction (B.L+D.S)is able to capture the secondary vortex at $\mathrm{x} / \mathrm{d}=5.5$. The Degani-Schiff corrections are specifically applied to capture the cross-flow separations better. But, the current implementation of compressibility corrections to SA model proposed by Catris and Aupoix (2000) does not use such corrections for cross-flow separation and are more general in nature and are used to account for large density gradients in the boundary layers. 
Figure 8 and Fig. 9 show the pitot pressure contours at the four sections for Case 1. A similar comparison for Case 2 is shown in Fig. 10 and Fig. 11. The figures indicate the cross-flow structure at four different $\mathrm{x} / \mathrm{d}$ locations for the two cases with and without the compressibility correction. The flow-field contours indicate the movement of vortex core away from the body as we move down stream. The shock associated with the cross flow separation is stronger for the original SA model. The primary vortex is much stronger with SA model.
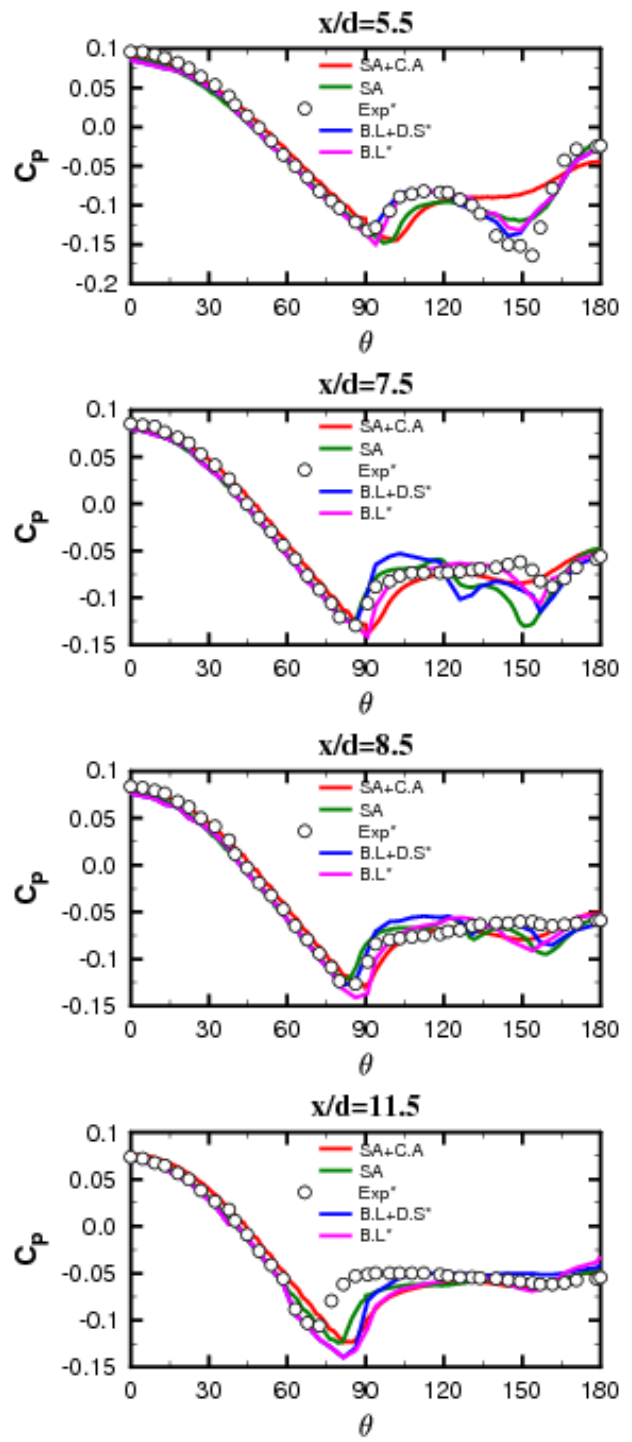

Fig. 6. Case 1: Comparison of present computed $C_{p}$ with experimental and computational results of Birch et al. (1994)*

Secondary separation and strong secondary vortices can also be noted for the original SA model. It can be noted that the flow structures are better defined for the original SA case. These structures are formed on the leeward side of the cylinder where the Mach numbers are lower. This indicates that the model with compressibility correction is more dissipative in low Mach number regions when compared to the original model. Figures 12 and 13 show density gradient contours at two axial locations for the cases considered.
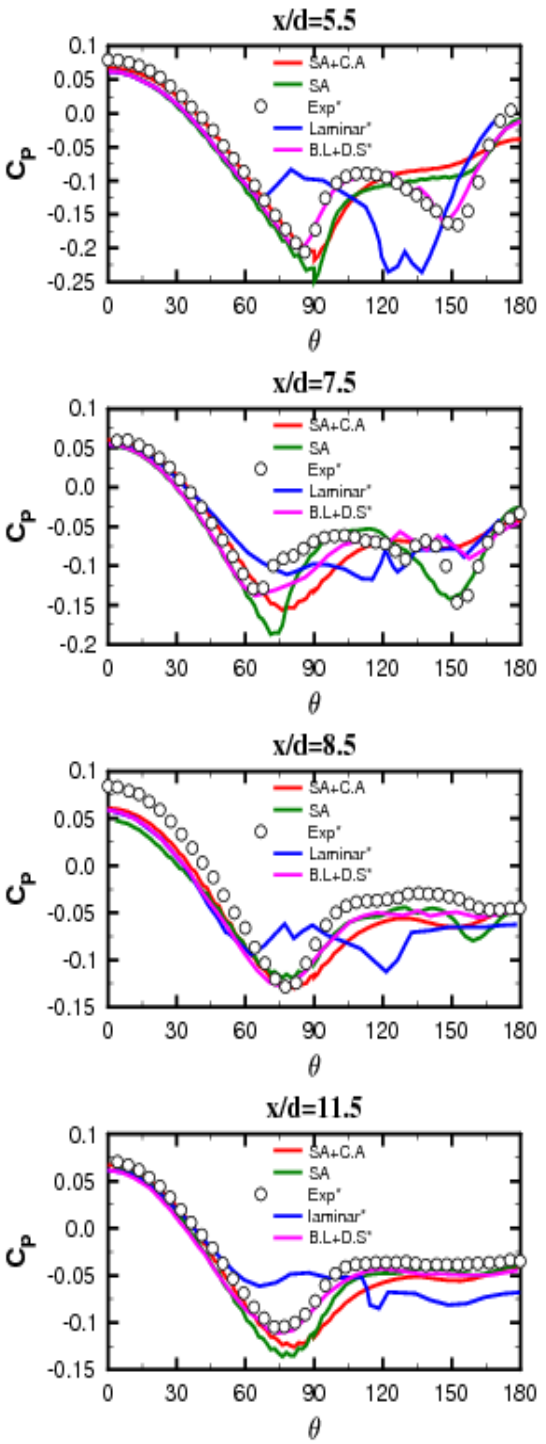

Fig.7. Case 2: Comparison of present computed $\mathrm{C}_{\mathrm{p}}$ with experimental and computational results of Sturek et al. $(1998)^{*}$

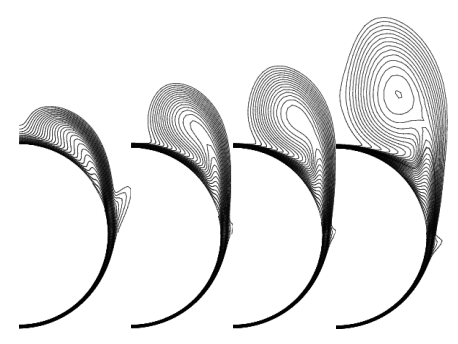

(a) $\mathrm{x} / \mathrm{d}=5.5$ (b) $\mathrm{x} / \mathrm{d}=7.5$ (c) $\mathrm{x} / \mathrm{d}=8.5$ (d) $\mathrm{x} / \mathrm{d}=11.5$ 
Fig. 8. Case 1: Pitot pressure contours $(\mathrm{SA}+\mathrm{CA})$

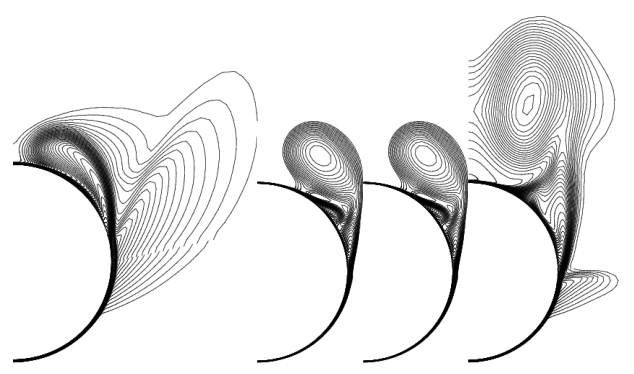

(a) $\mathrm{x} / \mathrm{d}=5.5$ (b) $\mathrm{x} / \mathrm{d}=7.5$ (c) $\mathrm{x} / \mathrm{d}=8.5$ (d) $\mathrm{x} / \mathrm{d}=11.5$

Fig. 9. Case 1: Pitot pressure contours (SA)

This figure shows the regions of high density gradients in the flow. When compared with the $\mathrm{C}_{\mathrm{p}}$ plots shown in Fig. 6 and Fig. 7, it can be noted that the compressibility corrected model performs better in these high density gradient zones.

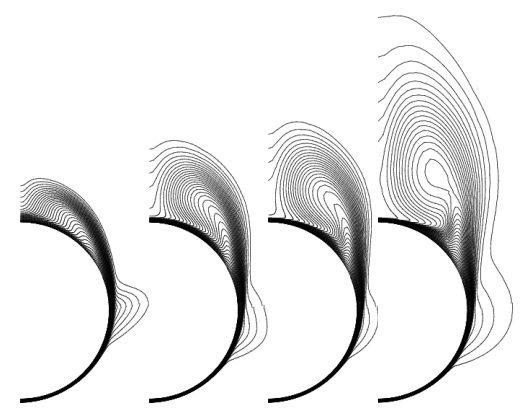

(a) $\mathrm{x} / \mathrm{d}=5.5$ (b) $\mathrm{x} / \mathrm{d}=7.5$ (c) $\mathrm{x} / \mathrm{d}=8.5$ (d) $\mathrm{x} / \mathrm{d}=11.5$

Fig. 10. Case 2: Pitot pressure contours $(\mathrm{SA}+\mathrm{CA})$

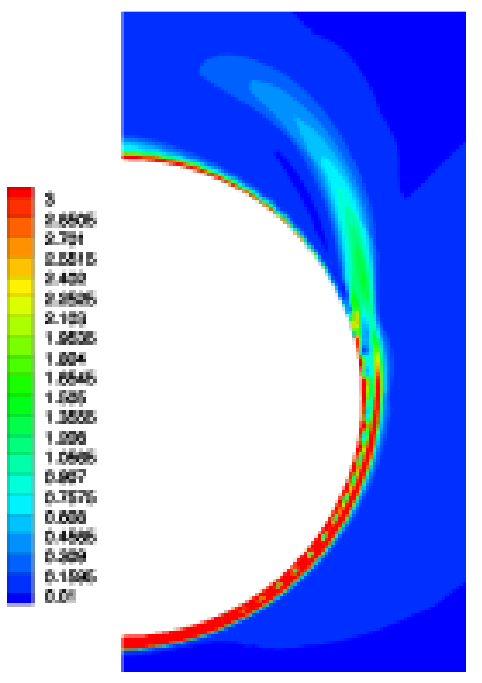

(a) $\mathrm{x} / \mathrm{d}=5.5$

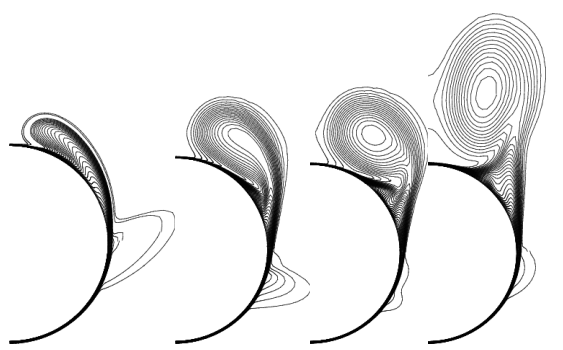

(a) $\mathrm{x} / \mathrm{d}=5.5$ (b) $\mathrm{x} / \mathrm{d}=7.5$ (c) $\mathrm{x} / \mathrm{d}=8.5$ (d) $\mathrm{x} / \mathrm{d}=11.5$

Fig. 11. Case 2: Pitot pressure contours (SA)

Volume streamlines for Case 1 and Case 2 are shown in Fig. 14 and Fig. 15, respectively. It can be noted that the vortex core is smaller and moves further away from the body for the SA model. The vortex remains closer to the body for SA+CA. This is also seen in the pitot pressure contours in Fig. 8 to Fig. 11.

Figure 16 shows $C_{p}$ vs. $x / d$ on leeward side of the body. For both the cases flow expands up to ogive- cylinder junction before separating by forming a shock. The strength of separation shock is more in the case of original SA computations compared to $\mathrm{SA}+\mathrm{CA}$ results. $\mathrm{C}_{\mathrm{p}}$ remains more or less constant for all the locations downstream of the junction for $\mathrm{SA}+\mathrm{CA}$ computations.

The normal and axial force coefficients for the two cases are shown in the Table 3. For the Case $1 \&$ Case 2 the present computed coefficient values were overpredicted compared to the original SA results and experimental results (Birch et al. 2000). 
N. Kumar and M. T. Nair / JAFM, Vol. 6, No. 3, pp. 375-384, 2013.

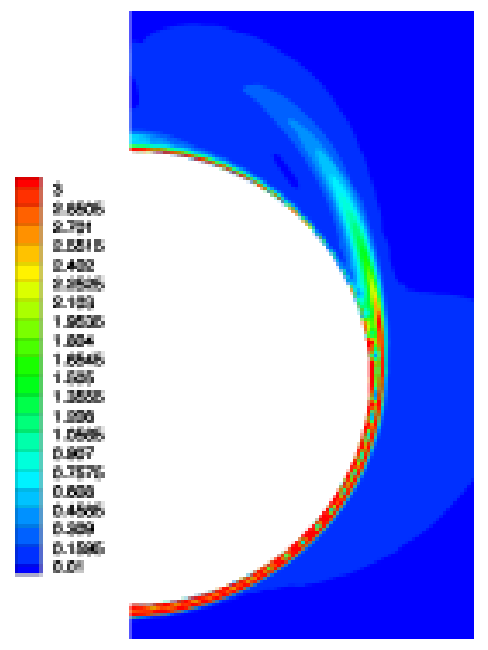

(a) $\mathrm{x} / \mathrm{d}=5.5$

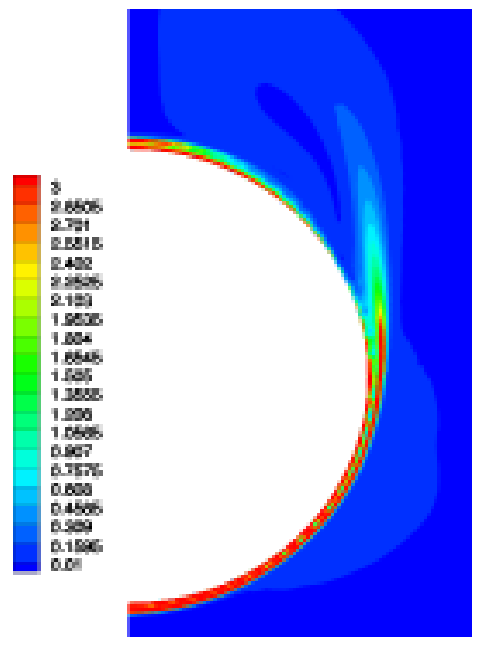

(b) $\mathrm{x} / \mathrm{d}=7.5$

Fig. 13. Case 2: Density gradient contours

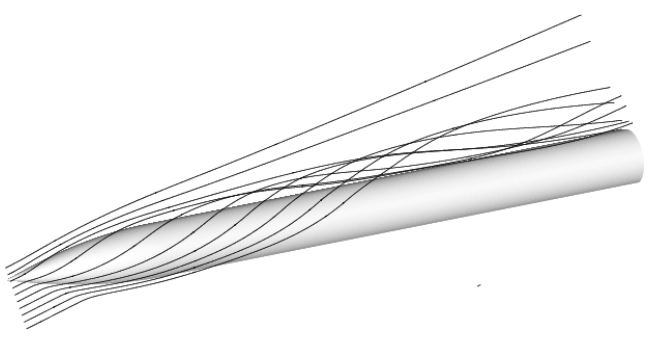

(a) $\mathrm{SA}+\mathrm{CA}$

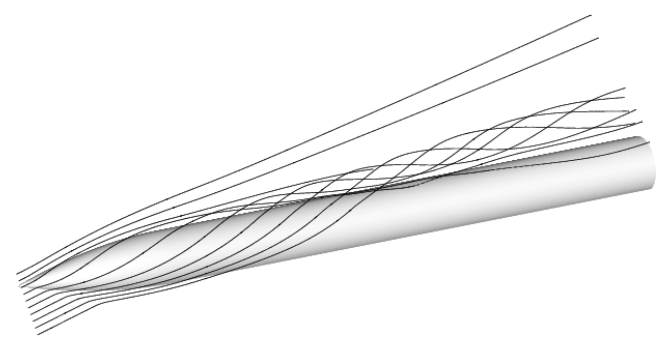

(b) SA

Fig. 14. Case 1: Volume Streamlines

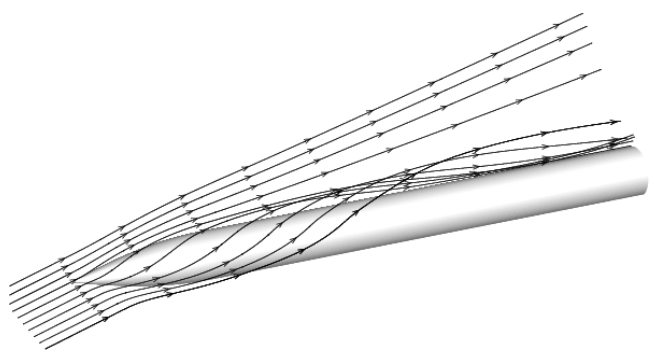

(a) $\mathrm{SA}+\mathrm{CA}$

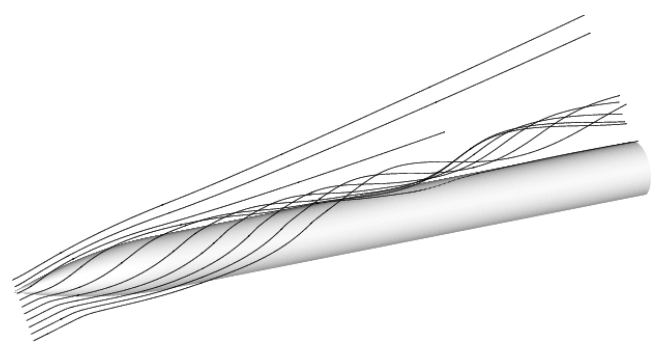

(b) SA

Fig. 15. Case 2: Volume Streamlines

Table 3 Comparison of present computed force coefficients with experimental values of Birch et al.(2000)

\begin{tabular}{|c|c|c|c|c|}
\hline Case & Coefficients & Exp & SA & SA+CA \\
\hline \multirow{2}{*}{1} & $\mathrm{C}_{\mathrm{a}}$ & 0.19 & 0.19 & 0.228 \\
\cline { 2 - 5 } & $\mathrm{C}_{\mathrm{n}}$ & 1.9 & 1.877 & 1.956 \\
\hline \multirow{2}{*}{2} & $\mathrm{C}_{\mathrm{a}}$ & - & 0.2 & 0.24 \\
\cline { 2 - 5 } & $\mathrm{C}_{\mathrm{n}}$ & - & 1.44 & 1.58 \\
\hline
\end{tabular}




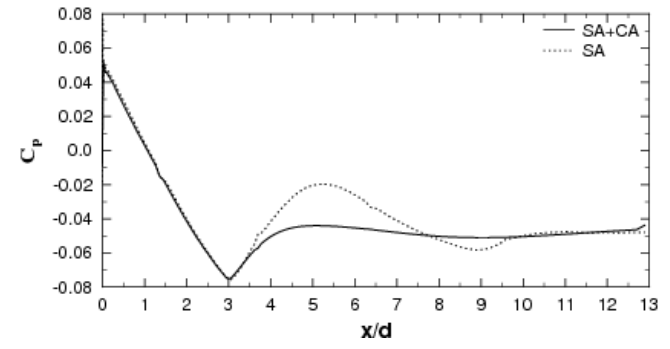

(a) Case 1: $M_{2}=2.5, n=14^{2}$

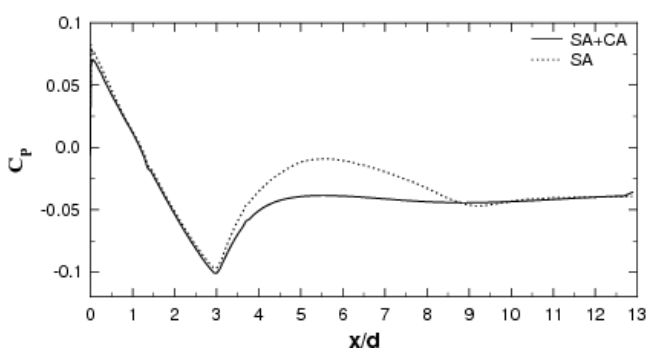

(b) Case 2: $M_{x}=1.8, \alpha=14^{\circ}$

Fig. 16. Case 1: $C_{p}$ distribution along length on the leeward side of the body

\section{Conclusion}

The aim of the present paper is to study the performance of Spalart- Allmaras model with density correction due to Catris and Aupoix (2000) for predicting flows at high angles-of-attack. The compressibility correction is used to make the model consistent with the logarithmic law of the compressible boundary layer in the presence of high density gradients. The model is tested for flow past an ogive cylinder at $14^{\circ}$ angle-of-attack. Two different Mach numbers are considered.

The results show that the corrected SA model is able to capture the overall flow features at high angles-ofattack. The $\mathrm{Cp}$ variations show that the $\mathrm{SA}+\mathrm{CA}$ model does better up to the separation point and is able to improve the prediction of the separation point. After the separation point the predictions are not very good. The primary and secondary vortices are weaker. The secondary vortex at $\mathrm{x} / \mathrm{d}=5.5$ is not captured. Therefore the corrected model is performing better in regions of density gradients and high Mach numbers. However, once the flow is separated and high density gradient and high Mach numbers are not present; the performance of the model deteriorates.

The flow features in the separated region are dissipative as seen in the pitot pressure plots and volume streamlines. The model is not able to capture the secondary flow in these regions. This reflects in the overall load predictions and the model predicts higher loads compared to the original model.

Overall it is observed that the corrected model performs well in regions of high Mach numbers and density gradients. A comparison of the performance of the present model with the original SA model indicates that the compressibility corrections should only act in regions of high density gradients in the boundary layer. This can be achieved by blending the two models..

\section{ACKNOWLEDGEMENTS}

This work has been supported by the ER\&IPR, DRDO, India under a Grants-in-Aid project.

\section{REFERENCES}

Baldwin, B. S. and T. J. Barth (1990). A one equation turbulence transport model for high Reynolds number wall bounded flows, NASA-TM 102847.

Baldwin, M. S. and H. Lomax (1978). Thin-layer approximation and algebraic model for separated flow, AIAA,78-257.

Birch, T. J., N. Qin and X. Jin (1994). Computation of supersonic viscous flows around a slender body at incidence, AIAA,94-1938.

Birch, T. J., I. E.Wrisdale and S. A. Prince (2000). CFD predictions of missile flowfields, AIAA,2000-4211.

Catris, S. and B. Aupoix (2000).Density corrections for turbulence models, Aerospace Science and Technology, 4, 1-11.

Deck, S., P. Duveau, P. d'Espiney and P. Guillen (2002). Development and application of SpalartAllmarasone equation turbulence model to threedimensionalsupersonic complex configuration, Aerospace Science and Technology, 6, 171- 183.

Degani, D. and L. Schiff(1986). Computation of turbulent supersonic flows around pointed bodies having cross flowseparation, Journal of Computational Physics, 66,173-196.

Gridgen (1999). User manual version 13.3, Pointwise.

Josyula, E. (1999). Computational simulation improvements of supersonic high angle-of-attack missile flows, Journal of Spacecraft and Rockets, 36 (1), 59-65.

Kumar, N., M. T. Nairand and S. K. Saxena (2004). Computation of supersonic viscous flow around a slender body at high angles-of-attack. In Proceedingsof the Tenth Asian Congress of Fluid Mechanics, University of Peradeniya, Sri Lanka.

Kumar, N., M. T. Nairand and S. K. Saxena (2005). Numerical simulation of high angle-of attack, aerodynamics.Computational Fluid Dynamics Journal, 13(4), 6, 58-68.

Hafez M. and K.Oshima, (Eds), 2, Word Scientific, Computational Fluid Dynamics Review, 734-745. 
N. Kumar and M. T. Nair / JAFM, Vol. 6, No. 3, pp. 375-384, 2013.

Roe, P. L. (1981). Approximate Riemannsolvers, parameter vectors, and differenceschemes. Journal of ComputationalPhysics, 43, 357-372.

Sarkar, S., G. Erlebacher, M. Hussainiand, H. Kreiss(1991). The Analysisand Modeling of Dilatational Terms in Compressible Turbulence. Journal of Fluid Mechanics, 227, 473-493.

Sarkar, S. (1992). The Pressure-Dilatation Correlation in Compressible Flows. Physics of Fluids A4(12), 2674-2682.

Saxena, S., K. and M. T. Nair (2002). Implementation and testing of SpalartAllmaras model in a multi-block code, AIAA,2002-0835.

Spalart, P. R. and S. R. Allmaras (1992). A one equation turbulence model for aerodynamic flows, AIAA,92-0439.
Sturek, W. B., D. Frist, M. Taylor, H. Thornburgand B.Soni(1998). Navier-stokes predictions of missile body separated flow fields.

Van Albada, G. D., B. Van Leer and W. W. Roberts, (1982). A comparative study of computational methods in cosmic gas dynamics. Astronomy and Astrophysics, 108, 76-84.

Yoon, S., and A. Jameson (1987). An LU-SSORscheme for the Euler and Navier-Stokes equations, AIAA, 87-0600.

Zeman, O. (1990). Dilatation Dissipation:The Concept and Application in ModelingCompressible Mixing Layer.Physics of Fluids A 2, 178-187.

Zeman, O. (1993). New Model for super/hypersonicturbulent boundary layers, AIAA,93-0897. 